\title{
KARATE - A Setup for High Rate Tests of the CMS Outer Tracker 2S Module Readout Chain
}

\author{
Alexander Dierlamm, Ulrich Husemann, Stefan Maier*, Thomas Müller \\ on behalf of the CMS Tracker Collaboration \\ Karlsruhe Institute of Technology - Institute of Experimental Particle Physics \\ E-mail: s.maierekit.edu
}

\begin{abstract}
KARATE (KArlsruhe high RAte TEst) is a new test system to stress test the readout chain of detector modules for the upgrade of the CMS Outer Tracker for the high-luminosity LHC. Modules consisting of two silicon strip sensors, called $2 \mathrm{~S}$ modules, are deployed in the outer regions of the Outer Tracker. The readout chain of a $2 \mathrm{~S}$ module consists of 16 CMS Binary Chips (CBC) each connected to two stacked silicon strip sensors. The CBC contributes data to the first trigger level by identifying particles with large transverse momenta. The output is sparsified on two concentrator chips which in turn are connected to a Gigabit transceiver that prepares the data for output through an optical module. Standard test systems such as test beams or radioactive source measurements need a track reconstruction or do have Gaussian distributed hit profiles and do not reach the occupancy or trigger rates expected in the future Outer Tracker of CMS. KARATE uses a combination of LEDs and photodiodes to inject hit patterns with varying pulse heights, occupancies and trigger rates into the front-end of the CBC, giving full control on 48 channels at $40 \mathrm{MHz}$. This offers the opportunity to directly compare injection patterns with readout patterns. This contribution introduces the test system and summarizes measurements on a CBC that is read out electrically.
\end{abstract}

Topical Workshop on Electronics for Particle Physics (TWEPP2019)

2-6 September 2019

Santiago de Compostela, Spain

\footnotetext{
${ }^{*}$ Corresponding Author.
} 


\section{Phase-II Upgrade of the CMS Tracker}

To exploit the physics potential of the High Luminosity LHC (HL-LHC) CMS will replace the entire tracking detector with an enhanced version by 2026 [1]. The Outer Tracker (OT) consists of so called $p_{T}$ modules [2]. The $p_{T}$ module type deployed in the outer region of the OT is called $2 \mathrm{~S}$ module. Each $2 \mathrm{~S}$ module consists of two closely spaced silicon strip sensors read out by the CMS Binary Chip (CBC) [3], which discriminates particles according to their transverse momentum by exploiting their bent trajectory in the CMS magnetic field. High $p_{T}$ particles cross the module in a less bent trajectory than low $p_{T}$ particles. The information of high $p_{T}$ particles contributes to the Level 1 trigger decision at $40 \mathrm{MHz}$. The CBCs are placed on a flex circuit hybrid which has a fold-over to connect the strips on both sensors. They are connected to Concentrator Integrated Circuits (CIC) [4] linked to a Low-power Gigabit Transceiver (LpGBT) [5]. The data is sent out via a Versatile Transceiver Plus module (VTRx+) [6]. This chain will have to cope with mean track densities (MTD) of up to $15 \mathrm{MHz} / \mathrm{cm}^{2}$ and trigger rates of up to $750 \mathrm{kHz}$, with up to 200 concurrent inelastic collisions (pile-up, PU). Tests such as measurements with radioactive sources or beam tests do not reach the occupancy and trigger rates expected in the OT. Nevertheless, the results obtained so far proved the $\mathrm{CBC}$ to work under low occupancy and trigger conditions [3]. A new test system was developed, called KArlsruhe high RAte TEst (KARATE), to stress test the readout chain under high occupancy and trigger rates.

\section{KArlsruhe high RAte TEst: KARATE}

Figure 1a shows a schematic of the KARATE system. Two FC7 boards [7] are used to emit light patterns and to readout a CBC hybrid. The first FC7 board controls 48 LEDs (650 nm) emitting light patterns routed via optical fibres onto photo diodes, which substitute strips of a silicon sensor. Each diode is wire-bonded to the input pad on the hybrid for a CBC channel. The second FC7 board reads out the $\mathrm{CBC}$ hybrid with an interface card. A connection between emitter and readout FC7 transmits the trigger signal and a $40 \mathrm{MHz}$ clock generator synchronises the FC7s. Future upgrades will extend the readout chain with a CIC, LpGBT and the VTRx.

(a)

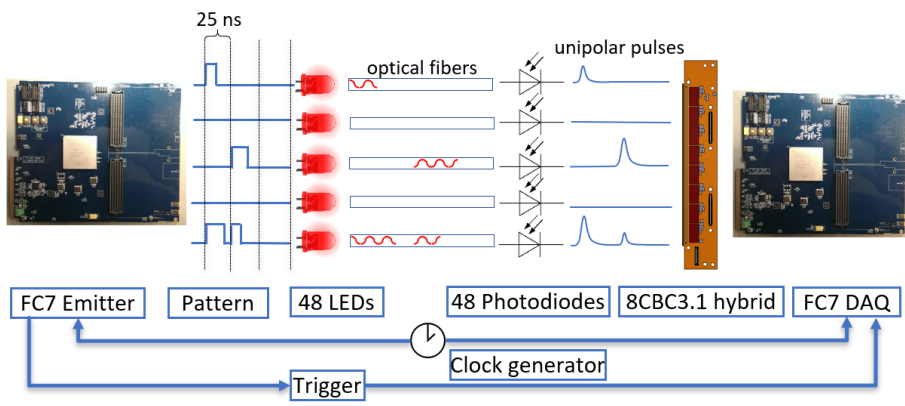

(b)

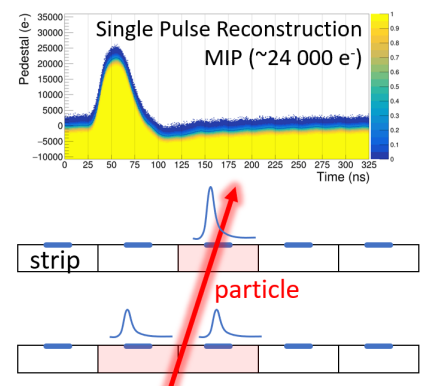

Figure 1: (a) In the KARATE system an FC7 controls 48 LEDs generating light routed via optical fibres on 48 photo diodes. Each diode is connected to a CBC channel. The CBCs are read out by an interface card connected to an FC7. (b) The injection emulates particles crossing two sensors, including charge sharing.

A C++-based framework generates patterns which are stored on the emitting FC7 and injected every $25 \mathrm{~ns}$. It compares the readout data with the injection patterns for a large variety of possible 
measurement scans. Each pattern contains information on the pulses to be injected and a trigger bit. If this bit is set, a trigger signal is sent to the readout FPGA simultaneously with the light emission.

The amplitude of a single pulse on the CBC front-end depends on the bias voltage applied on the LED and the light pulse duration. Higher bias voltages increase the light intensity resulting in larger pulse amplitudes for equal exposure times on the photo diode. The light pulse duration can be set from 0 to $46.875 \mathrm{~ns}$ in steps of $3.125 \mathrm{~ns}$. Therefore, pulses with 16 different amplitudes can be realised on the front-end for each channel in a pattern for a given bias voltage. With a calibration measurement the correlation between front-end signal and the light pulse length on the LED is obtained for each channel and bias voltage. As shown in Fig. 1b, the pulse amplitude can be reconstructed by scanning the occupancy while varying the threshold for different phase settings on the CBC's front-end.

The model used for pattern generation emulates particles crossing the two sensors assuming an active thickness of $290 \mu \mathrm{m}$, each. The trajectory inside the active volume is split in sections of $3 \mu \mathrm{m}$. A signal following a Landau distribution for particles trespassing $290 \mu \mathrm{m}$ of silicon is generated and then scaled to $3 \mu \mathrm{m}$ for each section. Charge sharing is considered by splitting the generated signal on the strips passed as shown in Fig. 1b. The calibration is used to inject a signal with a light pulse length for which the resulting front-end pulse is closest to the sum of all signals along the track sections assigned to this channel. By varying the most probable value of the Landau distribution, different irradiation levels resulting in differing cluster signals can be emulated.

\section{Cross-talk Measurements}

To evaluate the cross-talk between channels of the readout electronics single pulses with constant height are injected into one channel at $400 \mathrm{kHz}$. Using internal test pulses would not probe the effects of the signal propagation on the hybrid. The pulse on the injection channel and the baseline behaviour on its neighbours is reconstructed and then repeated with ten different pulse heights and on all channels.

(a)
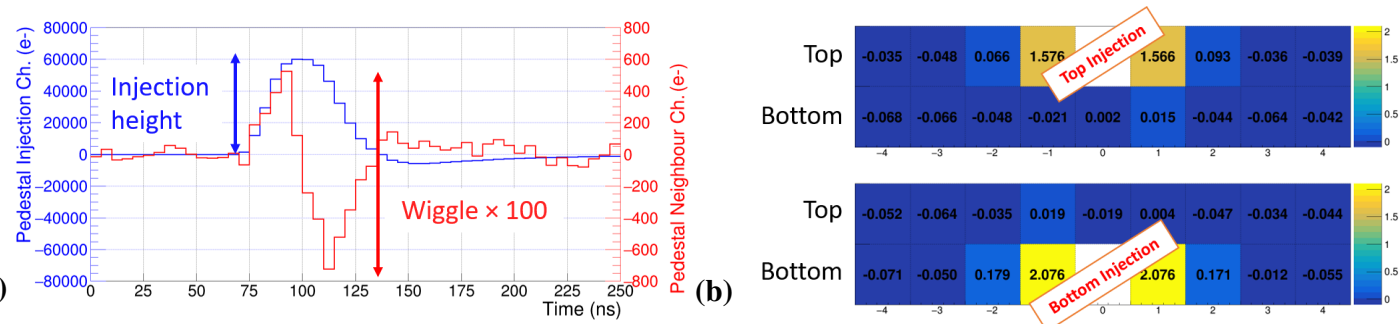

Figure 2: (a) A pulse injection in a single channel (blue) and its effect on a adjacent channel (red). (b) The cross-talk on adjacent channels for top channel and bottom channel injections.

As shown in Fig. 2a, the injection causes an oscillation (wiggle) on the neighbouring channels. The cross-talk is defined as the difference between the wiggle's maximum and minimum value with respect to the injected pulse height. As expected, the highest influence is seen in directly adjacent channels as shown in Fig. 2b. For an injection in a channel on the top-side of the hybrid the crosstalk is about $1.6 \%$, which is small compared to a bottom channel injection resulting in about $2.1 \%$. The difference is caused by the hybrid fold-over, resulting in longer signal lines of the bottom 
channels and thus a higher probability for cross-talk. The values obtained are not considered to impair the sensor readout.

\section{Trigger Rate Scans}

Depending on the instantaneous luminosity of the HL-LHC the mean PU varies between 140 and up to 200. The hit detection efficiency is scanned depending on the mean trigger and particle rate. Perpendicular tracks with varying MTDs between 10,15 and $20 \mathrm{MHz} / \mathrm{cm}^{2}$ emulate different module positions in the OT. The cluster signal is reduced to $17 \mathrm{ke}^{-}$emulating the radiation level of a sensor expected at the end of its operation. The injection happens at $40 \mathrm{MHz}$ while the trigger signal separation is Poisson-distributed with mean values varying between 80 and 45 bunch crossings. The efficiency of finding cluster signals is of most interest. A recorded cluster is valid if its centre of gravity is within 1.5 strips compared to the injection cluster. The threshold of the readout chip is set to $5 \mathrm{ke}^{-}$which is about five times a channel's noise. In Fig. 3a, the cluster efficiency is well above $99 \%$ for the whole mean trigger rate range and all different track densities. More important than the absolute efficiency is the fact that the efficiency is independent of the trigger rate.
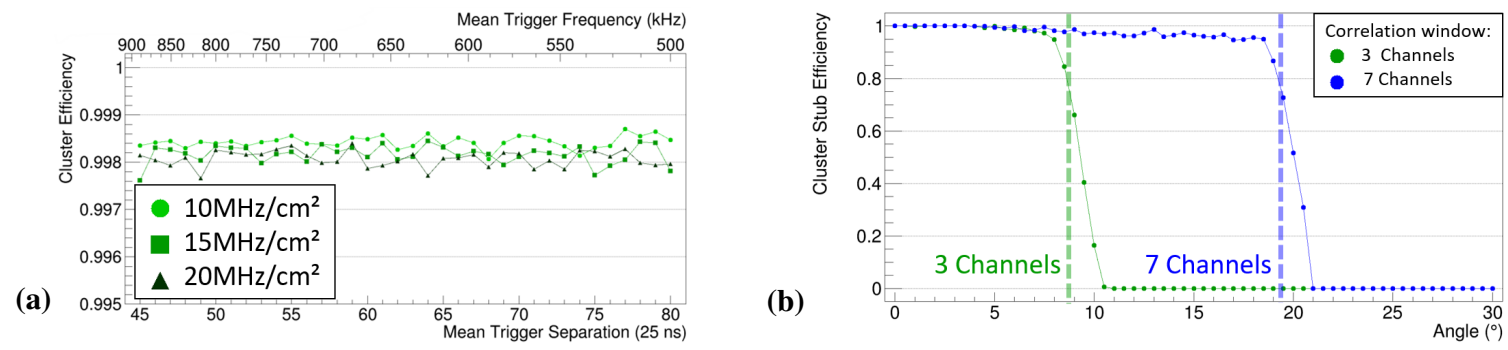

Figure 3: (a) The cluster efficiency depending on the mean trigger separation for different MTDs.

(b) The efficiency of finding stubs depending on the trajectory angle for different acceptance windows.

\section{Track Inclination and Stubs}

$p_{T}$ modules discriminate traversing particles on their transverse momentum. If the second sensor is hit within an adjustable correlation window, the information of the first hit's position and the bend value, the offset of the hit position on the second sensor, is sent out to the back-end. Patterns emulating tracks with different angles are injected at $40 \mathrm{MHz}$ while monitoring the stub efficiency. The triggers are time-separated according to a Poisson distribution with a mean value of 53 bunch crossings resulting in an average trigger rate of $750 \mathrm{kHz}$. Two different correlation window sizes are set on the CBC. As expected, there is a distinct drop in the efficiency of finding stubs that contain clusters within 1.5 strips of the injection clusters once the incidence angle surpasses the geometric acceptance window indicated in Fig. 3 b.

\section{Threshold Scans}

Each efficiency measurement depends on the threshold setting and the pulse height injected in the front-end. Figure 4a shows a threshold scan with two different conditions emulating the cluster signals of an unirradiated $\left(22 \mathrm{ke}^{-}\right)$and an irradiated $\left(17 \mathrm{ke}^{-}\right)$silicon sensor. The results of the signal emulating an unirradiated sensor match a ${ }^{90} \mathrm{Sr}$ source measurement very well. Minimal 
ionizing particles, as they are emitted by ${ }^{90} \mathrm{Sr}$, deposit a cluster signal of about $22 \mathrm{ke}^{-}$in $290 \mu \mathrm{m}$ of active silicon. The measurement also compares two PU scenarios. The first one emulates a MTD of $10 \mathrm{MHz} / \mathrm{cm}^{2}$ and a PU of 140 . The resulting mean trigger rate is $500 \mathrm{kHz}$. The second emulates a MTD of $15 \mathrm{MHz} / \mathrm{cm}^{2}$ resulting in a mean PU of 200 and a mean trigger rate of $750 \mathrm{kHz}$. The independence of the cluster efficiency with respect to the MTD and trigger rate is clearly visible. The measurement shown in Fig. 4b confirms a high cluster finding efficiency even at high trigger rates and low cluster signals, as they are expected after ten years of operation.

(a)
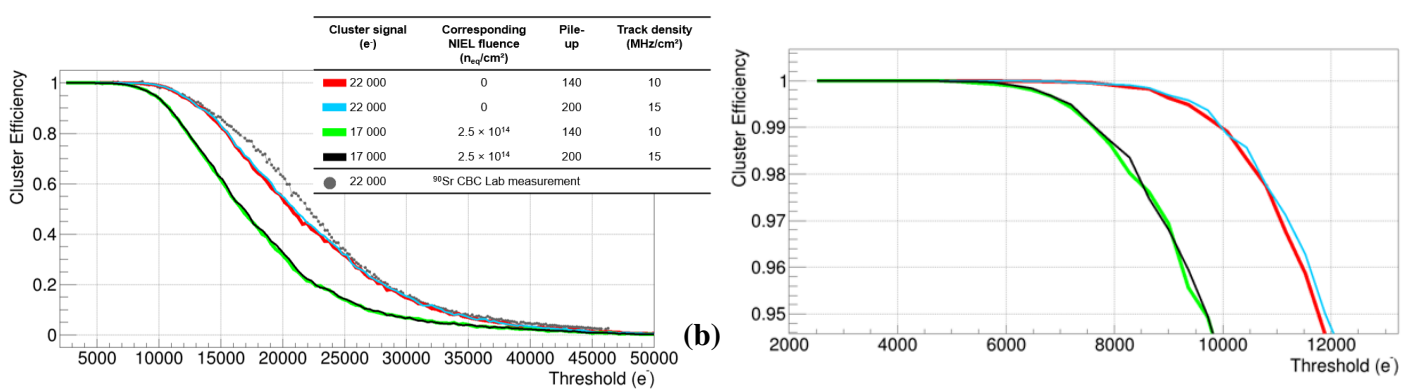

Figure 4: (a) The cluster efficiency depending on the threshold for two cluster signals and MTDs compared to a laboratory-based ${ }^{90} \mathrm{Sr}$ measurement. (b) Enlarged view on the cluster efficiency for low thresholds.

\section{Conclusion and Outlook}

The KARATE system confirms the high rate functionality of the CBC under various conditions such as trigger rate, threshold and stub scans. The readout chain will be extended as soon as more final components will become available.

\section{References}

[1] CERN. The HL-LHC project. June 16, 2019. URL: http://hilumilhc.web.cern.ch/.

[2] CMS Collaboration. The Phase-2 Upgrade of the CMS Tracker. Tech. rep. CERN LHCC-2017-009. CMS-TDR-014. CERN, Apr. 16, 2018. URL: https://cds.cern.ch/record/2272264.

[3] K. Uchida et al. The CBC3 readout ASIC for CMS 2S-modules. Tech. rep. CMS-CR-2018-017. Geneva: CERN, 2018. URL: https://cds.cern.ch/record/2312215.

[4] B. Nodari et al. A $65 \mathrm{~nm}$ Data Concentration ASIC for the CMS Outer Tracker Detector Upgrade at HL-LHC. Tech. rep. CMS-CR-2018-278. CERN, Oct. 2018. URL: https://cds.cern.ch/record/2650712

[5] The Low power GBT Team. LpGBT Specifications. Ed. by P. Moreira. 2018. URL: https://espace.cern.ch/GBT-Project/LpGBT/Specifications/LpGbtxSpecifications.pdf.

[6] J. Troska et al. The VTRX+, an optical link module for data transmission at HL-LHC. In: PoS TWEPP-17 (2017), 048. 5 p. DOI: 10.22323/1.313.0048.

[7] M. Pesaresi et al. The FC7 AMC for generic DAQ control applications in CMS. In: JINST 10.03 (2015), p. C03036. DOI:10.1088/1748-0221/10/03/C03036 